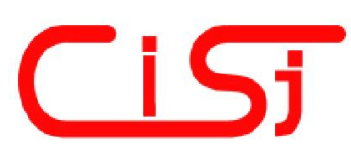

\title{
APPLICATION OF NUMERICAL INTELLIGENCE METHODS FOR THE AUTOMATIC QUALITY GRADING OF AN EMBRYO DEVELOPMENT
}

\author{
Domas Jonaitis, Vidas Raudonis, Arunas Lipnickas \\ Department of Automation, Kaunas University of Technology, Lithuania \\ domas.jonaitis@ktu.edu, vidas.raudonis@ktu.lt, arunas.lipnickas@ktu.lt
}

\begin{abstract}
In vitro fertilization - a procedure which aims to get the embryo to adapt the methods of "oocyte" fertilized sperm outside the human body. At the end of this procedure there are several embryos. This paper represents overview of tracking-free and tracking-based methods for detection of important embryo development stages. Tracking-based method represents well known classical object tracking techniques. For tracking-free method were selected statistical feature extraction techniques and classification methods: Classification with training and classification without training. For the feature extraction proposed statistical methods: entropy, invariant moments and principal components analyses. For the classification are used neural networks, support vector machine and K-nearest neighbor method. Data collected consist of 500 images for each class. 70 percent of images are dedicated for training, and 30 percent for testing. The proposed method is checked by experiment. It is expected that this method will work well in video sequences. Copyright (C) Research Institute for Intelligent Computer Systems, 2016. All rights reserved.
\end{abstract}

Keywords: In vitro fertilization, computer vision, classification.

\section{INTRODUCTION}

In vitro fertilization is one assisted reproductive technology. The aim of this procedure is to get the embryo to adapt the methods of "oocyte" fertilized sperm outside the human body. There are several embryos at the end of this procedure. So it is highly delicate question how to choose the best embryo to be transferred to the uterus. The researchers have been proposed a variety of techniques which deals with a proper embryo selection question. In the Fig. 1 there is shown, the stages of embryo development.

In this paper there is introduced the automatic embryo development method which can assist embryologist during most important embryo selection task [1]. The newest way to track embryo development is time-lapse microscopy. This is an automatic image capturing method, when the computer finds and determine fetal position. In the Fig. 1 highlighted the critical times between the stages of embryo development, which is one of the main features, that can predict successful development of human embryo. One of the most popular methods is the early nucleus fission time [3]. Early embryo development is the process, when the cell of nucleus divides into four cells. Nucleus fission time moments is one of the most important features by which can be made a decision to choose embryo for transfer.

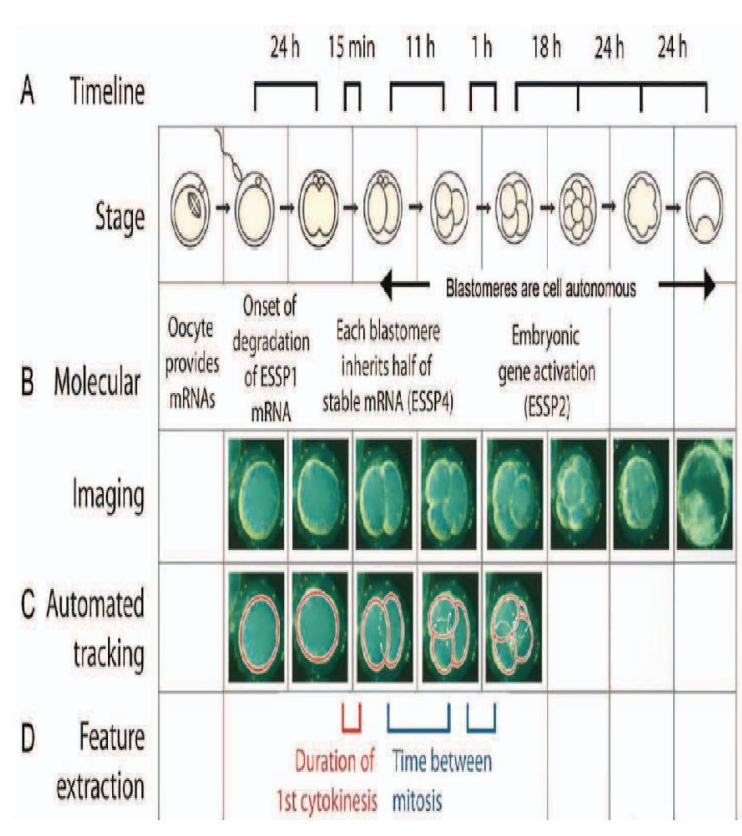

Fig. 1 - Images of embryo development. Figure reproduced with permission from Wang et al. [2]

Therefore, there are more features by which decision can be made, such as thickness of embryo coat, fragmentation level, symmetry of nucleus.

Purpose of this article is to compare some methods, to detect the fission moments of embryo.

This research article is further divided into following sections: 1) Computer vision methods; 
2) methodology; 3) experimental data; 4) results; 5) conclusions.

\section{COMPUTER VISION METHODS}

Time lapse and image analysis systems are used for assisted reproduction about 25 years. Current commercial time -lapse systems are tracking from 14 to 84 or more embryos at a time. So there are many data to proceed. The automated time lapse systems, has to detect the embryo, and to develop their quality grade.

The computer vision based methods which detects an early embryo cleavage can be classified into two major groups:

a) tracking-based;

b) tracking free methods.

Tracking based method applies computer vision algorithms which detects the contours of the embryo cells, measures an area of each individual cell and tracks them during the whole development process [16].

Tracking-free methods uses low level features extracted from time-lapse embryo images which corresponds certain cleavage stage of the embryo. The combination of low level images features forms certain recognizable which are almost similar from the stage to stage.

Tracking-based methods are based on an assumption that the embryo itself and cells from which consist the embryo have clear or elliptical shape. There for, the embryo detection task is simplified to detection to known or predictable number of ellipses in time-lapse image. Ellipse detection can be established using reduced quantization-free parameters space [4] or by analyzing of principal components [5]. Basically, three stable points are needed to fit an ellipse on the object contour [6-8]. Meanwhile the accuracy of ellipse detection highly depends on accurate detection of the object contour which is a difficult task in many cases dealing with embryo images.

Tracking - free methods uses feature extraction techniques, which can be grouped in four groups: First group appearance based, which incorporates well statistical methods: PCA, LDA, ICA and others, Feature based approach computes Gabor features, Binary features and others. Template based uses certain image samples which are compared with real time images. Part-based approaches compute local features of the object. Feature extraction techniques such as Sift, Surf, FAST, ORB, BRIEF computes stable and discriminating features which are invariant to rotation and uneven illumination. All mentioned tracking-free methods highly depends on the training samples, from which features are extracted. The training sample should represent all possible variation of the traceable object.

\section{EXPERIMENTAL SETUP AND THEORETICAL BACKGROUND}

\subsection{EXPERIMENTAL SETUP:}

The purpose of the experimental investigation is efficiency evaluation of the proposed classification algorithm that is used for recognition of different embryo stages. Therefore, 3 different feature extraction methods and 3 different classification algorithms are tested using labeled data sets.

The principal component analysis (PCA), entropy, and $H u$ invariant moments are used as a feature extraction methods in the research. The classification is done using support vector machine (SVM), artificial neural networks (ANN) and K-nearest neighbor algorithms. Mentioned classification algorithms are well known methods and commonly used for similar tasks. Basically, classification algorithms strongly depend on the correct training data set, which have to be correctly labeled and sorted. Freely available database of labeled embryo images where used in the investigation [9]. All images were labeled by the experience embryologist. Available images were divided evenly (50/50) in two groups, i.e., training and testing data sets. Data base consist of the image of the embryos of four different stages. First stage or class represents an embryo which consists of one cell (see Fig. 9). Second set of images represents an embryo which consists of two cells and it is labeled as second class (see Fig. 10). Third developmental stage of the embryo is considered when the embryo consist of three cells and all images which records that are labeled as third class (see Fig. 11). Fourth class is when the embryo consists of four cells (see Fig. 12).

All programming, modeling and experimental investigation were executed using MATLAB programming environment.

\subsection{THEORETICAL BACKGROUND:}

The principal component analysis (PCA), entropy, and $H u$ invariant moments are used as feature extraction methods. All images can be statistically measured and measurements can vary depending on the information that is captured in those images. One such statistical measure is entropy. This is measure that represents an energy levels decoded in the image. If an image has flat and even surface it will have zero entropy. If there exists huge variation in the pixel values the entropy value will be high. The entropy [10] function was applied on the images from database which can be expressed as follow: 


$$
\text { Entropy }=-\sum_{i} P_{i} \log _{2} P_{i}
$$

where, $\mathrm{P}_{\mathrm{i}}$ is the probability that the difference between 2 adjacent pixels is equal to $i$, and $\log _{2}$ is the base 2 algorithm.

$H u$ invariant moments are another statistical measure which are used for image measurements [11]. Hu moments are translation invariant and can be normalized with respect to changes in scale and rotation. Seven $\mathrm{Hu}$ moments were used as inputs for ANN, SVM and kNN classification algorithms. These were used in a simple pattern recognition experiment to successfully identify various typed characters. They are computed from normalized centralized moments up to order three and are shown below:

$$
\begin{gathered}
I_{1}=\prod_{20}+\eta_{02} \\
I_{2}=\left(\eta_{20}-\eta_{02}\right)^{2}+4 \eta_{11}^{2} \\
I_{3}=\left(\prod_{30}-3 \eta_{12}\right)^{2}+\left(3 \eta_{21}+\eta_{03}\right)^{2}
\end{gathered}
$$

$$
I_{4}=\left(\eta_{30}+3 \eta_{12}\right)^{2}+\left(\eta_{21}+\eta_{03}\right)^{2}
$$

$$
\begin{gathered}
I_{5}=\left(\eta_{30^{-}} 3 \eta_{12}\right)\left(\eta_{30}+\eta_{12}\right)\left[\left(\eta_{30}+\right.\right. \\
\left.\left.\eta_{12}\right)^{2}-3\left(\eta_{21}+\eta_{03}\right)^{2}\right]+\left(3 \eta_{21}-\eta_{03}\right)( \\
\left.\eta_{21}+\eta_{03}\right)\left[3\left(\eta_{30}+\eta_{12}\right)^{2}-\left(\eta_{21}+\eta_{03}\right)^{2}\right]
\end{gathered}
$$

$$
\begin{aligned}
& I_{6}=\left(\eta_{20}-\eta_{122}\right)\left[\left(\eta_{30}+\eta_{12}\right)^{2}-\left(\eta_{21}+\right.\right. \\
& \left.\left.\eta_{03}\right)^{2}+4 \eta_{11}\left(\eta_{30}+\eta_{12}\right)\left(\eta_{21}+\eta_{03}\right)\right]
\end{aligned}
$$

$$
\begin{aligned}
I_{7}= & \left(3 \eta_{21}-\eta_{02}\right)\left(\eta_{30}+\eta_{12}\right)\left[\left(\eta_{30}+\eta_{12}\right)^{2}-\right. \\
& \left.3\left(\eta_{21}+\eta_{03}\right)^{2}\right]+\left(\eta_{30}-3 \eta_{12}\right)\left(\eta_{21}\right. \\
& \left.+\eta_{03}\right)\left[3\left(\eta_{30}+\eta_{12}\right)^{2}-\left(\eta_{21}+\eta_{03}\right)^{2}\right]
\end{aligned}
$$

Principal component analysis (PCA) is a statistical procedure that uses an orthogonal transformation to convert a set of observations of possibly correlated variables into a set of values of linearly uncorrelated variables called principal components [12]. The number of principal components and projections depends of the amount of data that will be used for classification.

PCA rotates the original data space such that the axes of the new coordinate system point into the directions of highest variance of the data. Performing principal component analysis directly on the embryo images is computationally costly. Therefore, the size of the covariance matrix is limited by the size $l$ of the training data set, because 1 usually much smaller than the amount of pixels in the image. PCA is obtained from the eigen- decomposition of a covariance matrix and these eigenvectors can be estimated if matrix $\mathrm{C}$ satisfies the eigenvalue equation:

$$
\mathrm{Cv}=\lambda v
$$

where, the eigenvalue $\lambda$ and eigenvector $v$ is associated to square covariance matrix C. The estimated eigenvalues allows ranking the eigenvectors according to their usefulness to describe the data variation between training images.

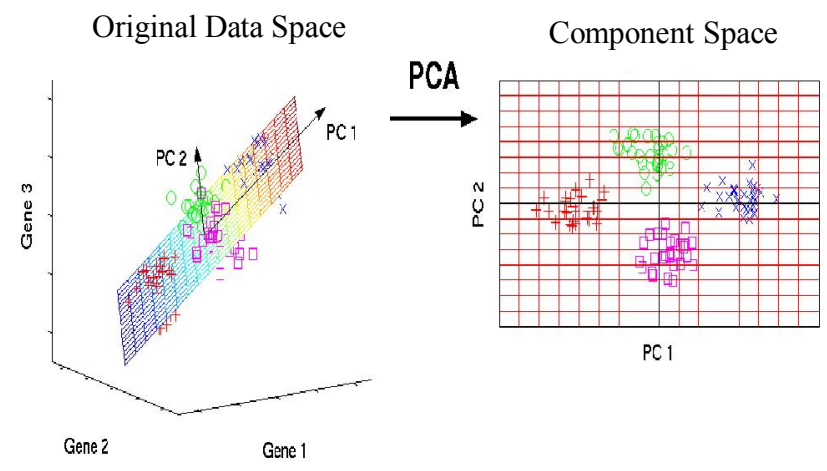

Fig. 2 - Principal component analysis

Eigenvalues can be estimated using equation:

$$
\operatorname{det}(C-\lambda I)=0
$$

where, $\mathbf{I}$ is an image.

Artificial neural network was used for the classification purposes, where the number of input is varying depending on chosen number of classification features. For classification, neural network [13] is used, which output can be subscribed:

$$
y=\varphi\left(\sum_{j=0}^{n} w_{j} x_{j}\right)
$$

where, $(\mathrm{x} 0, \mathrm{x} 1, \mathrm{x} 2, \ldots, \mathrm{xn})$ - inputs, $(\mathrm{y})-$ Output, $\mathrm{i}-$ the weights of inputs and the function $\varphi$-activation function.

Feed forward neural network is used for this research. Levenberg - Marquardt backpropagation training algorithm is selected.

Logarithmic sigmoid transfer functions was selected, and ten hidden layer and linear transfer function in the output layer of the model. For the input 16 PCA coefficients were selected, also entropy and invariant moments values.

Other classification method is K-nearest neighbor algorithm [14]. This method is used to compare the results with neural network and support vector machine. K-nearest neighbor algorithm similarity score is calculated by the following formula: 


$$
s\left(d, c_{j}\right)=\sum_{d_{i} \in k-N N} \operatorname{sim}\left(d, d_{i}\right) y\left(d_{i}, c_{j}\right)
$$

where $\operatorname{sim}\left(d, d_{i}\right)$ the similarity between image $d$ and class $d_{i}$.

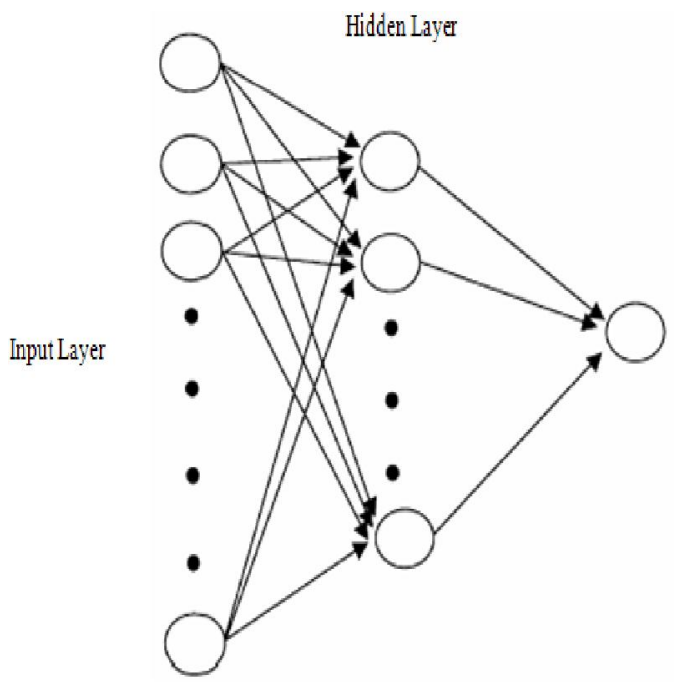

OutputLayer

Fig. 3 - Neural network

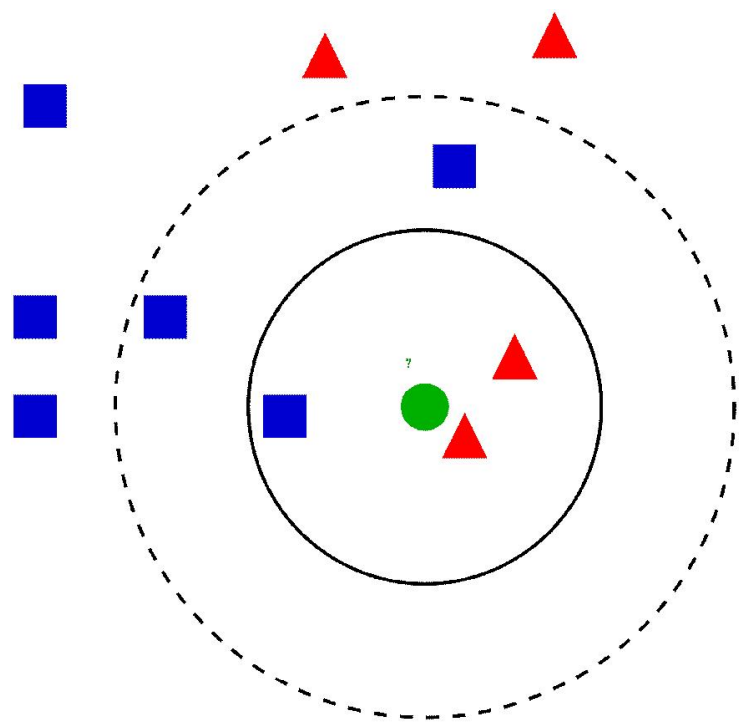

Fig. 4 - K-nearest neighbour algorithm

Support Vector Machines are one of the more popular and powerful "off-the-shelf" machinelearning algorithms based on their ability to find non-linear patterns. SVMs work by finding a line, known as a "decision boundary" or "hyperplane", that best separates your data based on the class (in our case, "bullish" or "bearish" bars).[15]The SVM then draws a line in the higher dimensional space that maximizes the distance between the two classes. When a new data point is presented to the SVM, it then calculates which side of the line the point falls and makes its prediction. So in this research all classes separately were compared with all data.

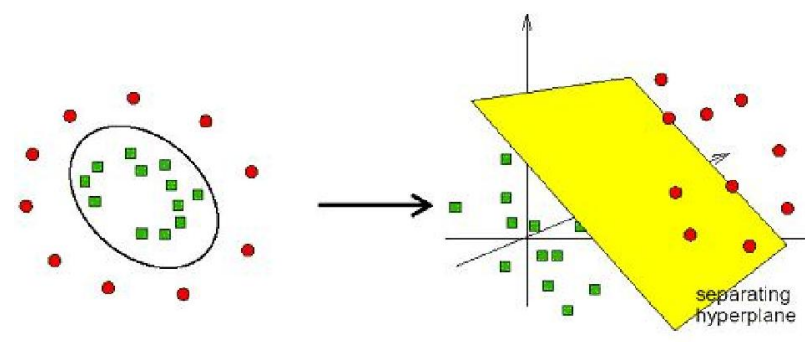

complex in low dimensions

simple in higher dimensions

Fig. 5 - Support vector machine

\section{ANALYSES AND RESULTS}

The analysis of this research can be divided into two groups: Tracking based [16] and tracking fee. First analysis was to investigate the ability to detect the embryo. In the fig 6 represented the classical background subtraction method. This method is very useful because for example from Fig. $5 \mathrm{a}$ it is possible to find the thickness of the pellucid zone. It is enough just to count radial gray pixel variation from the center.

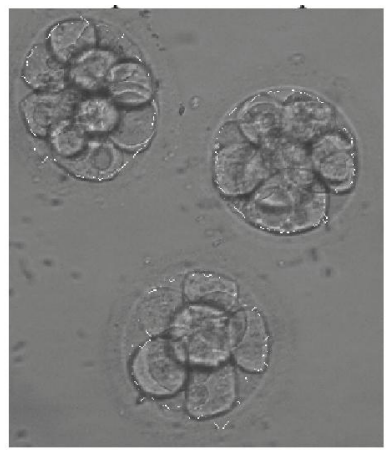

a)

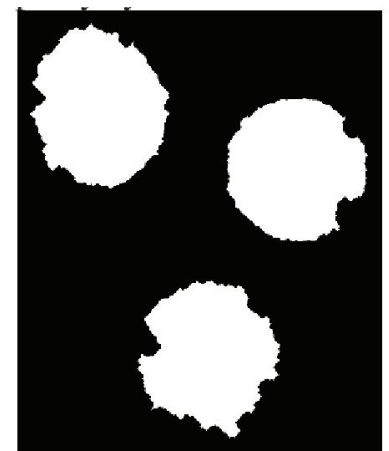

b)
Fig. 6 - The 3 embryos (a), boundaries of the cells (b) [16]

The kurtosis and skewness of grey values shows the shell radius beginning and the end Fig. 7.

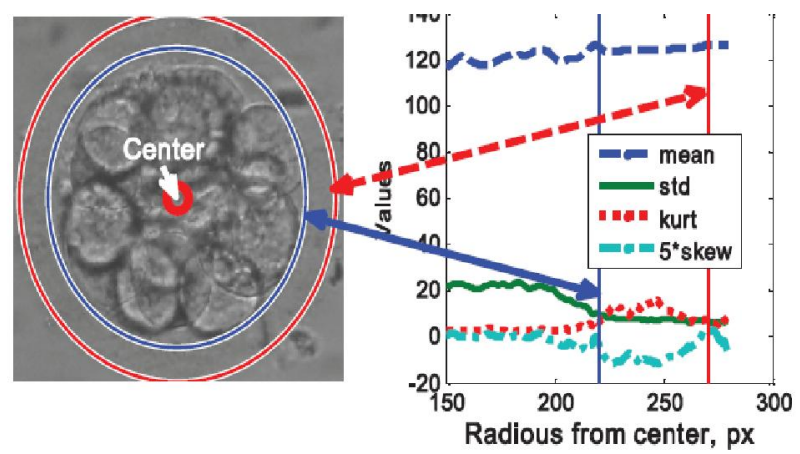

Fig. 7 - Detection of embryo pellucid zone

Also it is enough easy to extract the cells using various "filt" type filters. First need to filter the image, and after the fitting the ellipse to the filtered image is not a trivial task. Just need to adjust 
parameters. The difficult things appear just when cells are overlapped Fig. 8(b).

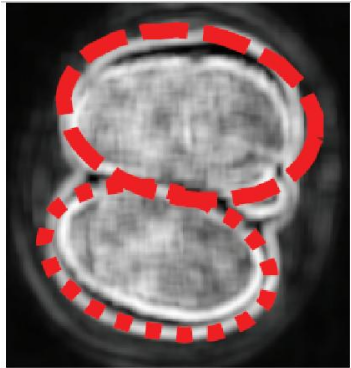

a)

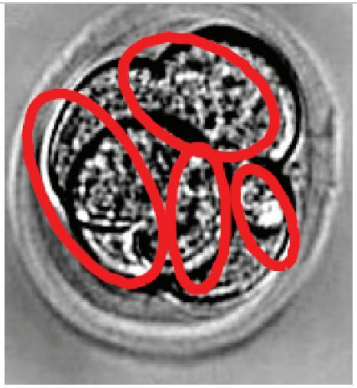

b)
Fig. 8 - Ellipse fitting to embryo cells: a - 2cells and $b-4$ cells $[16]$

Second analysis of this research was to adapt classification methods. For this research we selected data of 2000 images (500 for each class). Data were collected from free available database [9] (Fig. 912).
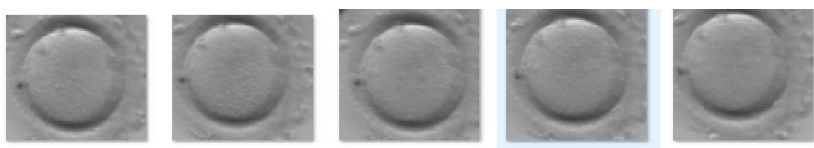

Fig. 9 - One cell of embryo
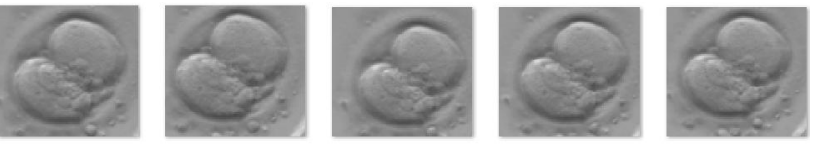

Fig. 10 - Two cells of embryo
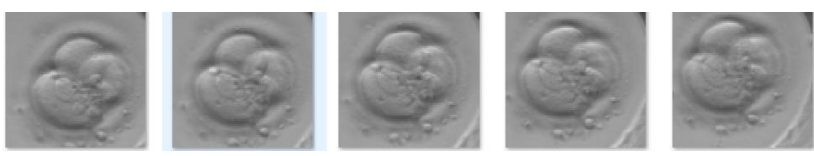

Fig. 11 - Three cells of embryo
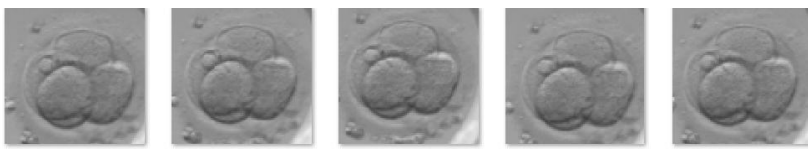

Fig. 12 - Four cells of embryo

In first stage of this research the features were extracted. The examples of extracted features submitted bellow:

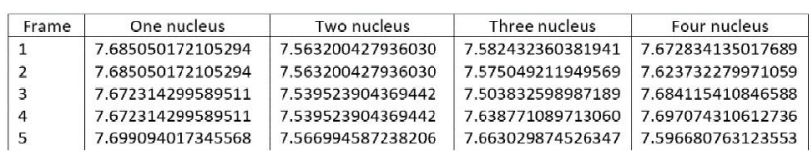

Fig. 13 - The entropy values fragments of each class

\begin{tabular}{|c|c|c|c|c|}
\hline Frame & One nucleus & Two nucleus & Three nucleus & Four nucleus \\
\hline \multirow{15}{*}{1} & 0.966528724530410 & -4.976067178381150 & -2.801559613736563 & 0.608240912646916 \\
\hline & 2.101065473429166 & -0.184059373418128 & -0.374099638491891 & 1.454199899734550 \\
\hline & 0.243029624857310 & -0.009289334005829 & -0.069916477658810 & -0.523261179385246 \\
\hline & 0.333735312207404 & 0.175145928932713 & 0.069407364828431 & -0.130874024256887 \\
\hline & -0.045148905965369 & 0.074732608555364 & 0.051067194280684 & 0.019438945638558 \\
\hline & -0.180562402568506 & 0.092201878370941 & 0.097982836064425 & $\begin{array}{l}0.6369110428901 / 3 \\
-0.429825764071519\end{array}$ \\
\hline & 1.211690372874501 & 0.774217347483406 & 0.053188691233873 & 0.376901846622636 \\
\hline & -0.291711394440801 & 0.138230708903851 & 0.039142313347159 & -0.061297715215545 \\
\hline & -0.574417670144986 & -0.098035839617935 & 0.047409957050647 & 0.174465080383044 \\
\hline & -0.445910863117401 & 0.141094411113032 & 0.194267217377151 & 0.766869000146533 \\
\hline & -0.183026272293181 & 0.263460218726084 & 0.019681578185135 & -0.040356129940736 \\
\hline & -0.116932051192127 & 0.121223791460348 & -0.105155918897446 & -0.193918812112015 \\
\hline & -0.104844973663114 & -0.195455632577580 & -0.111081563115564 & 0.090087515777589 \\
\hline & -0.127360773428061 & -0.183406826304695 & -0.054576968865706 & $\begin{array}{l}0.301221691408267 \\
0.608240912649616\end{array}$ \\
\hline & 0.306970697822589 & -0.187375805876724 & 0.028480425357250 & \\
\hline
\end{tabular}

Fig. 14 - The 15 PCA coeficients, of each class

\begin{tabular}{|c|l|l|l|l|l|}
\hline Frame & \multicolumn{2}{|l|}{ One nucleus } & Two nucleus & Three nucleus & Four nucleus \\
\hline \multirow{4}{*}{1} & 0.011809285952778 & 0.715789580830763 & 0.541892700503943 & 0.017508635885359 \\
& -0.000000000004348 & 0.000065939651944 & -0.000066922196473 & -0.000000000004552 \\
& -0.000000135158625 & -0.007322968793837 & -0.004684607951774 & 0.000000366135545 \\
& -0.000000008450029 & -0.011021435750441 & -0.029380757248270 & -0.000000065971995 \\
& 0.000842706502081 & 2.082254229279224 & 1.390216515885090 & 0.001693328230776 \\
& 0.000003380613805 & 0.518683805916161 & 0.266048550801067 & 0.000009361741859 \\
& 0.000765830073433 & 2.358928475400965 & 1.449300937135317 & 0.001605519861746 \\
\hline \multirow{6}{*}{2} & 0.011809285952778 & 0.715789580830763 & 0.498380827841830 & 0.627777856786567 \\
& -0.000000000004348 & 0.000065939651944 & -0.000076604641878 & -0.001279291812172 \\
& -0.000000135158625 & -0.007322968793837 & 0.001953769197919 & 0.002663743396875 \\
& -0.000000008450029 & -0.011021435750441 & -0.042091795469229 & -0.050275933568072 \\
& 0.000842706502081 & 2.082254229279224 & 1.262763934032163 & 1.986631941279850 \\
& 0.0000033800613805 & 0.518683805916161 & 0.236951727889957 & 0.401795113191295 \\
& 0.000765830073433 & 2.358928475400965 & 1.275578386157658 & 1.990133521011410 \\
\hline
\end{tabular}

Fig. 15 - Invariant moments of each class

The classification results were investigated with 3 classifiers using principal component analysis. The results in Fig. 16 showed that best precise of classification about $88 \%$ were got with support machine classificatory. The experiment showed that the best classification results were obtained when 16 PCA coefficients were used. The different numbers of PCA coefficients are presented in the $\mathrm{x}$ axis in Fig. 16. This experiment was made for selecting the optimal number of PCA coefficients. In the Fig. 16, we can see the difference of accuracy using different number of PCA coefficients.

\section{Clasification results using PCA}

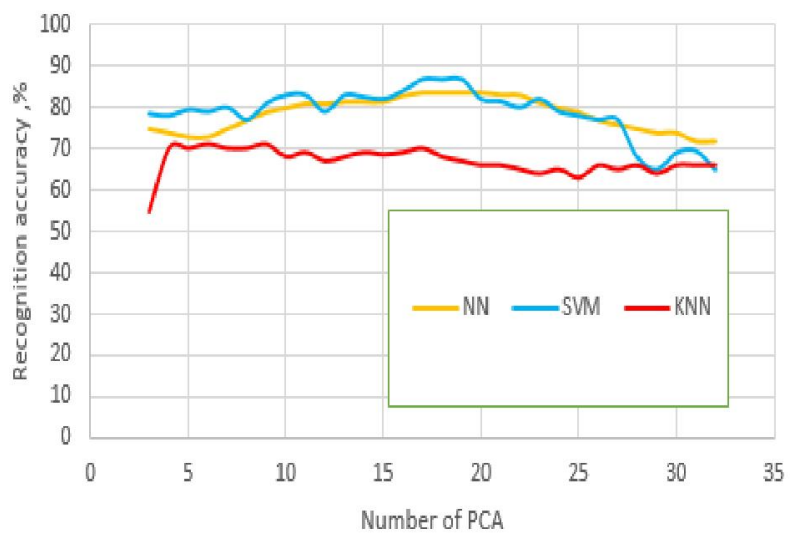

Fig. 16 - Classification results with different classifiers using PCA

Second experiment was made using all in section "methodology" mentioned feature extractions 
methods. The best result using support vector machine, and neural networks was got using 16 PCA coefficients, entropy value and 7 invariant moments all together as inputs for classifiers. If more PCA coefficients are used, the accuracy drops down. Invariant moments and entropy also increase the rate of accuracy. For neural network 10 hidden layers were selected. KNN best results were got using invariant moments, entropy and 9 PCA coefficients. If we have more features, the accuracy drops down. (Fig. 17) In the $x$ axis are represented 7 invariant moments + entropy value + PCA coefficients. This test represents the feature influence to the rate of accuracy.

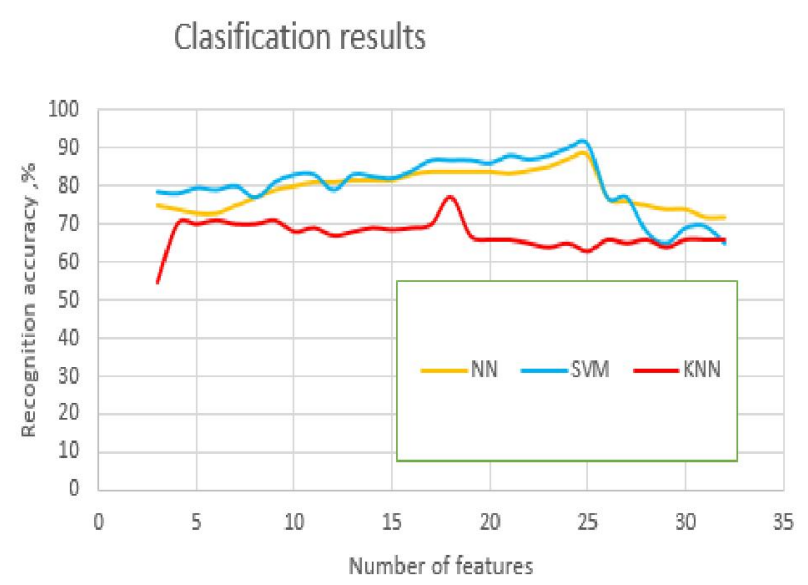

Fig. 17 - The results of classification

Above mentioned results, were got using average of all 4 cells, which were used for this experiment. For separate class the results are different. The best results using all three classifiers were got, when we have one cell. Then the accuracy is about $90 \%$. The accuracy goes down, then we have to detect 4 cells. It is falling to $80 \%$.

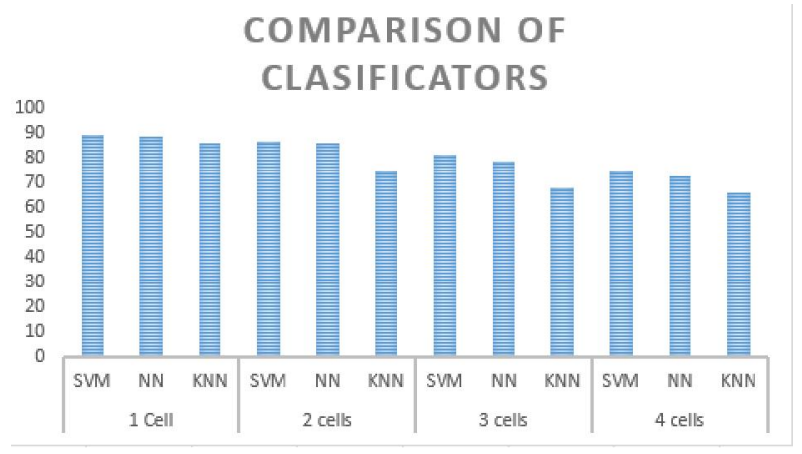

Fig. 18 - The accuracy of classification for each class separately

\section{CONCLUSION}

This approach is still not well explored. Relevance of the topic is based on the statistics of infertile couples. With such a large number of infertile couples (one in eight couple has fertility problems), this method has practical and scientific relevance.

Tracking based methods is useful to detect embryo, and to track it. Also, it is enough good tool to detect 1 or 2 cells. When we have more cells, and it is overlapping this method is not useful.

Tracking free methods are enough good for this issue. Introduced methods enough well classifies such data. SVM classificatory classifies the data in average $86 \%$ precise, and it is enough good result. Neural Network algorithm common recognition average was $83.75 \%$, K-nearest neighbor algorithm, a common recognition average was $82.13 \%$. It is enough good rate for investigation of this method in real environment.

\section{REFERENCES}

[1] Jonaitis D., Raudonis V., "Automatic detection and tracking of embryo development," in Proceedings of the Conference on Biomedical Engineering, Kaunas, 2014, pp. 139-143.

[2] C. C. Wong, K. Loewke, N. Bossert, B. Behr, C. J. De Jonge, T. Baer, R. Reijo Pera, "Noninvasive imaging of human embryos before embryonic genome activation predicts development to the blastocyst stage," Nature Biotechnology, Vol. 28, Issue 10, pp. 11151121, 2010.

[3] Wharf E., Dimitrakopoulos A., Khalaf Y., Pickering S., "Early embryo development is an indicator of implantation potential," Reproductive Biomedicine Online, Vol. 8, No. 2, pp. 212-218, 2004.

[4] K. C. Chen, N. Bouguila, and D. Ziou, "Quantization-free parameter space reduction in ellipse detection," Expert Syst. Appl., Vol. 38, No. 6, pp. 7622-7632, 2011.

[5] H. Jiandong, "Ellipse detection based on principal components analysis," in Proceedings of the International Conference on Computer Application and system Modeling, 2010, pp. 258-261.

[6] J. K. Lee, B. A. Wood and T. S. Newman, "Very fats ellipse detection using GPU-based RHT," in Proceeding of the 19th IEEE International Conference on Pattern Recognition (ICPR'20008), Tampa, USA, 8-10 December 2008, pp. 1-4.

[7] W. D. Prasad and M. Leung, "Clustering of ellipses based on their distinctiveness: An aid to ellipse detection algorithm," in Proceedings of the 3rd IEEE International Conference (ICCSIT'2010), Chenda, China, 9-10 July 2010, Vol. 8, pp. 292-297. 
[8] T. Cooke, "A fast automatic ellipse detection," in Proceedings of the International Conference on Digital Image Computing: Techniques and Applications (Dicta'2010), Sydney, Australia, 1-3 December 2010, pp. 575-580.

[9] http://www.resolve.org/about/fast-facts-aboutfertility.html. [Accessed 2016-05-10].

[10] Hu M. K., "Visual Pattern Recognition by Moment Invariants," IRE Trans. Info. Theory, Vol. IT-8, pp. 179-187, 1962.

[11] Jolliffe I. T., Principal Component Analysis, Series: Springer, 2nd ed., Springer, NY, 2002, XXIX, $487 \mathrm{p}$.

[12] Fukushima K., "Neocognitron: A selforganizing neural network model for a mechanism of pattern recognition unaffected by shift in position," Biological Cybernetics, Vol. 36, Issue 4, pp. 93-202, 1980.

[13] Coomans D., Massart D. L., "Alternative knearest neighbour rules in supervised pattern recognition: Part 1. k-Nearest neighbour classification by using alternative voting rules," Analytica Chimica Acta, Vol. 136, pp. 15-27, 1982.

[14] Cortes C., Vapnik V. "Support-vector networks," Machine Learning, Vol. 20, Issue 3, pp. 273-297, 1995.

[15] D. Jonaitis, V. Raudonis, A. Lipnickas, "Application of computer vision methods in automatic analysis of embryo development," in Proceedings of the 8th International Conference on Intelligent Data Acquisition and Advanced Computing Systems: Technology and Applications (IDAACS'2015), Warsaw, Poland, 24-26 September 2015, Vol. 1, pp. 257-260.

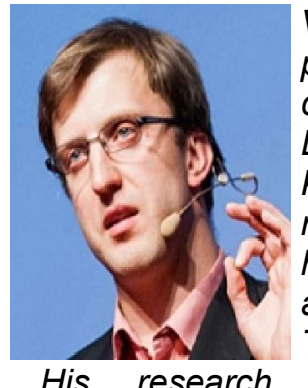

Vidas Raudonis is associate professor of automation and control system engineering at Department of Automation, Kaunas University of Technology, Lithuania. He obtained his PhD in Control Engineering at Kaunas University of Technology.

His research interest includes numerical intelligence, computer vision methods.

He participated in various research projects as supervisor.

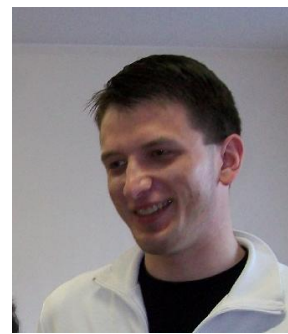

Domas Jonaitis is an informatics engineering $P h D$ student at Department of Automation, Kaunas University of Technology, Lithuania (supervisor. dr. Vidas Raudonis). He obtained his M. Sc. diploma with honors in Control Technologies (Intelligent Control Systems) in year of 2015.

PhD research topic is numerical intelligence methods for quality grading of embryo development.

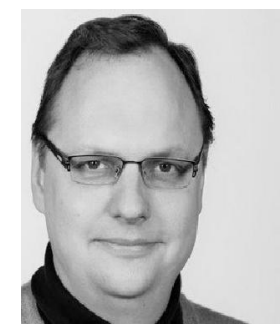

Arunas Lipnickas is a professsor of automation and control system engineering at Department of Automation, Kaunas University of Technology, Lithuania. He obtained his PhD in Control Engineering at Kaunas University of Technology.

His research interest includes robotics, intelligent systems, computer vision, advanced modeling, optimization and control system techniques with application in Technical systems. He participated in various research projects with international automation companies as senior researcher or supervisor. 